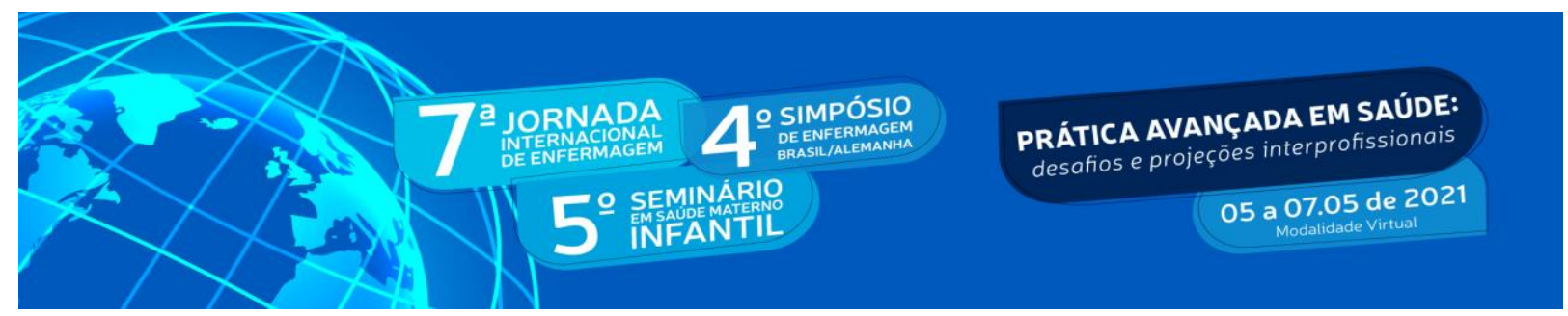

DOI: http://doi.org/10.48195/jie2021-173

\title{
PROCESSO DE ENFERMAGEM EM CENTRO OBSTÉTRICO: UMA REVISÃO NARRATIVA ${ }^{1}$
}

\section{Caroline Santini Rauber²; Bruna de Oliveira Jochims ${ }^{3}$; Evelyne Duarte de Amorim Silva $^{4}$; Fabricio da Cunha Moraes ${ }^{5}$}

\begin{abstract}
RESUMO
A assistência de enfermagem prestada às gestantes exige atuação direta e muito presente, a cada dia torna-se mais importante a utilização do processo de enfermagem (PE) durante o cuidado às essas pacientes. $\mathrm{O}$ trabalho teve por objetivo realizar uma revisão bibliográfica de estudos que mostrem como é realizado o PE em centro obstétrico (CO) no Brasil e identificar os sentimentos dos profissionais em relação à sua utilização. Este estudo foi desenvolvido por meio do levantamento de dados encontrados na literatura já existente. O PE é importante em CO, pois, proporciona a melhora na assistência às gestantes e puérperas. Os profissionais reconhecem essa importância, entretando, apontam dificuldades em sua realização, entre elas está a falta de conhecimento, falta de apoio da gestão, inexistência de treinamentos, sobrecarga no trabalho, defict de funcionários e pouca experiência. Grupo de estudo e treinamentos foram apontados como algumas das sugestões para a melhora da prática.
\end{abstract}

Palavras-chave: Processo de Enfermagem; Cuidados de Enfermagem; Avaliação em Enfermagem; Gestantes; Enfermagem Obstétrica.

\begin{abstract}
The nursing care provided to pregnant women requires direct and very present action, and the use of the nursing process (NP) during the care of these patients becomes more important every day. The objective of this study was to perform a bibliographic review of studies showing how the NP is carried out in the obstetric center (OC) in Brazil and to identify the feelings of professionals regarding its use. This study was developed through a survey of data found in the existing literature. The EP is important in OC because it provides improved care for pregnant and postpartum women. The professionals recognize this importance, however, they point out difficulties in its realization, among them are the lack of knowledge, lack of management support, lack of training, work overload, defict of employees and little experience. Study groups and training were pointed out as some of the suggestions to improve the practice.
\end{abstract}

Key Words: Nursing Process; Nursing Care; Nursing Assessment; Pregnant Women; Obstetric Nursing.

\footnotetext{
${ }^{1}$ Revisão de Literatura

${ }^{2}$ Enfermeira. Mestranda do Programa de Mestrado Profissional em Enfermagem. Relator. Universidade Federal de Ciências da Saúde de Porto Alegre. Porto Alegre, RS, Brasil. E-mail: caroline-santini@ hotmail.com

${ }^{3}$ Enfermeira. Coautor. Hospital Santa Casa de Misericórdia de Porto Alegre. Porto Alegre, RS, Brasil. E-mail: brujochms@hotmail.com

${ }^{4}$ Enfermeira. Coautor. Hospital Santa Casa de Misericórdia de Porto Alegre. Porto Alegre, RS, Brasil. E-mail: evelyneduarte.silva@gmail.com

${ }^{5}$ Enfermeiro Supervisor do Centro Cirúrgico Obstétrico. Coautor. Hospital Santa Casa de Misericórdia de Porto Alegre. Porto Alegre, RS, Brasil. E-mail: fabricio.enfo@gmail.com
} 


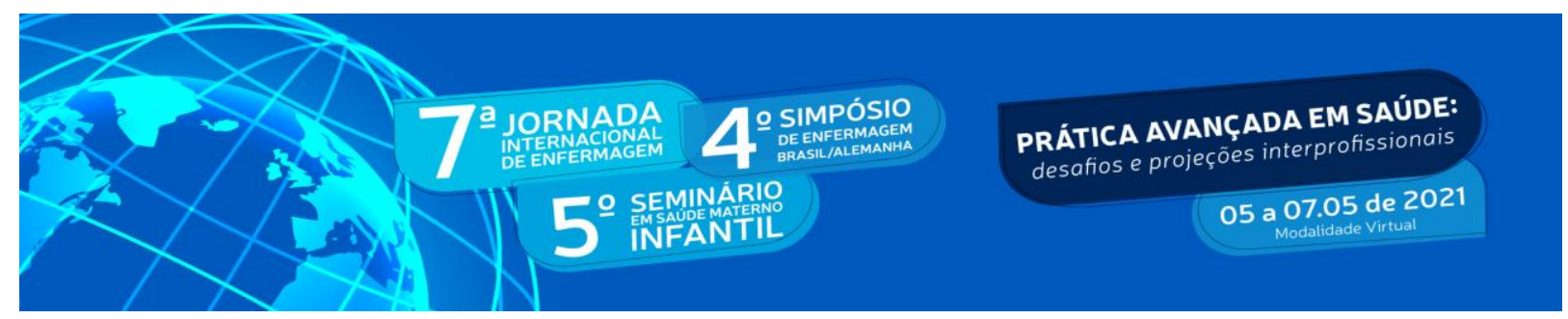

\section{INTRODUÇÃ̃O}

A sistematização da assistência de enfermagem (SAE) é uma metodologia desenvolvida a partir do conhecimento prévio do enfermeiro (a) e da sua prática a fim de garantir o cuidado integral e individualizado ao paciente. O SAE utiliza 5 etapas que direcionam o processo de enfermagem (PE) para implantar ações específicas para promoção, prevenção, recuperação e reabilitação da saúde do sujeito e das pessoas ao seu redor (COREN, 2016). Esse processo de sistematização da assistência exige do profissional um conhecimento aprofundado da clínica do paciente, além de raciocínio crítico para que possa elencar quais as principais ações serão direcionadas para um caso específico a fim de promover o bem-estar e a qualidade da assistência prestada pela equipe de enfermagem.

Por se tratar de uma sistematização da assistência, o SAE é desenvolvido a partir de cinco etapas, sendo elas: a coleta de dados de enfermagem, diagnóstico de enfermagem, planejamento de enfermagem, implementação e avaliação de enfermagem que são "interrelacionadas, interdependentes e recorrentes" e que fazem parte do PE, segundo o COFEN, (2009). O enfermeiro é o profissional responsável pela supervisão da implementação e avaliação do PE nos serviços onde ocorre o cuidado profissional de enfermagem, e lhe é privativo a realização da etapa de diagnóstico de enfermagem e prescrição das ações desenvolvidas pela equipe de enfermagem.

O PE caracteriza-se por ações dinâmicas que tem como finalidade prestar assistência às pessoas, por meio de atos que se relacionam e coordenam entre si (HORTA, 1979). É necessário o pensamento de que, mesmo sem problemas aparentes, o enfermeiro (a) deve ser capaz de pensar além do bem-estar físico do indivíduo, parentes e/ou comunidade, sendo capaz de identificar fatores de risco que possam ser modificados para favorecimento da promoção e prevenção em saúde. Dessa forma o PE direciona a assistência dos profissionais de enfermagem e auxilia para a documentação das atividades desenvolvidas e orientadas por meio de embasamento teórico-científico, sendo assim, tais ações contribuem e refletem para uma maior notoriedade e valorização da profissão (COFEN, 2009).

Por existir essa associação do cuidado na assistência com achados científicos, o PE acaba sendo uma ferramenta importante que auxilia na acreditação hospitalar, está 


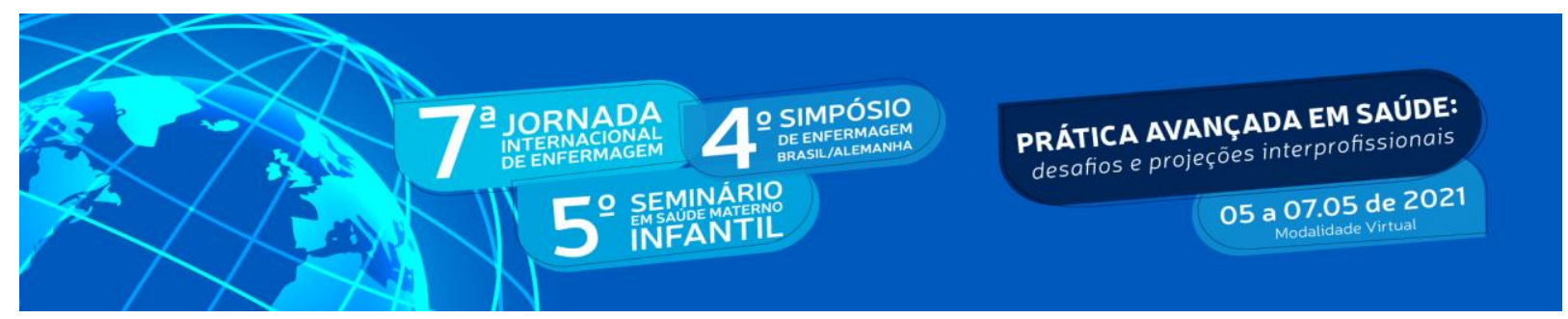

diretamente relacionado à segurança do paciente e qualificação da assistência. Portanto, é fundamental que os enfermeiros não utilizem esse instrumento apenas como algo rotineiro, de forma automática, sem que haja reflexão diante das atividades e situações vivenciadas (DIAS; DURAN, 2018).

Diante do exposto, durante a assistência exercida pela equipe, observa-se que a enfermagem exerce um papel importante para as gestantes, sendo este cuidado direto e muito presente. Porém, é presente a falha nos processos e na SAE, dificultada pela demanda elevada e muitas vezes por falta de conhecimento da equipe sobre a importância do processo de enfermagem em si.

Sendo assim, a utilização processo de enfermagem e aperfeiçoamento dos registros realizados pela equipe, trariam maior qualidade no cuidado prestado às gestantes em $\mathrm{CO}$, tornando mais segura a assistência de enfermagem o que faz com que a experiência das mulheres nesse processo seja a mais digna possível.

\section{OBJETIVO}

Identificar a produção científica acerca do processo de enfermagem em Centros Obstétricos.

\section{METODOLOGIA}

Estudo de revisão bibliográfica que objetiva a síntese de resultados sobre o tema do processo de enfermagem em centro obstétrico, de forma a possibilitar a incorporação de evidências na prática de enfermagem. Desta forma, foi realizado um levantamento de informações e busca ativa de documentos e artigos científicos em bibliotecas virtuais e três base de dados eletrônicas internacionais sendo elas: US National Library of Medicine National Institutes of Health (PubMed); a Literatura Latino-Americana e do Caribe em Ciência e Saúde (LILACS) e a biblioteca virtual Scientific Library Online (SciELO) por 


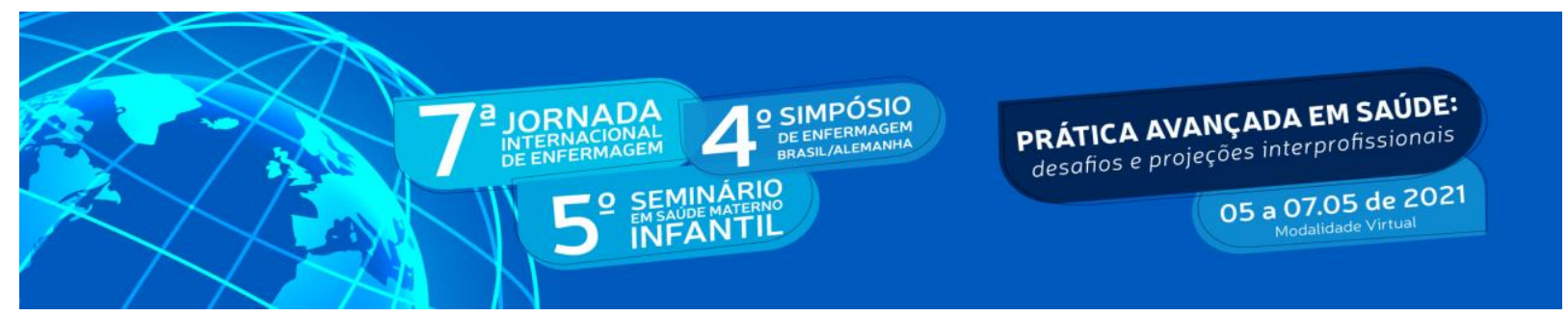

serem bases de dados amplas, com riqueza de conteúdos e apresentarem elevado rigor científico dos estudos.

Foram utilizados os cruzamentos dos seguintes Descritores em Ciências da Saúde (DeCS, versão 2020), em português e inglês: processo de enfermagem, cuidados de enfermagem, avaliação em enfermagem, gestantes, obstetrícia, salas de parto e enfermagem obstétrica, para realizar as buscas na literatura.

Foram critérios de inclusão para os artigos: data de publicação entre janeiro de 2012 e dezembro de 2020; nacionais ou internacionais; de enfermagem; redigidos em português ou inglês; artigos originais resultantes de pesquisas originais dos tipos quantitativa ou qualitativas, completos, de acesso livre online ou com resumos indexados nas bases de dados utilizadas. Foram excluídos artigos que não se adequaram aos critérios de inclusão e que não responderam a questão norteadora deste estudo. Os dados foram coletados entre fevereiro e março de 2021.

Para registro, análise e avaliação dos dados extraídos dos artigos utilizou-se um instrumento para a coleta de dados de revisões bibliográficas, estruturado em itens destinado ao registro das seguintes informações: número, título, autores, ano de publicação, periódico, local de publicação, objetivo, metodologia (tipo de estudo, campo das pesquisas/contexto, população/amostra, coleta de dados/informações), considerações relativas acerca do processo de enfermagem em centros obstétricos. A análise dos dados se deu através da releitura das informações contidas no instrumento elaborado para essa revisão bibliográfica e sua comparação com manuais técnicos.

\section{RESULTADOS E DISCUSSÃO}

No estudo de Medeiros et al. (2016), foram encontrados registros de 16 diagnósticos de enfermagem específicos para o trabalho de parto e parto. Estes diagnósticos estão relacionados às necessidades da parturiente e alterações advindas da gestação. Em relação às mulheres em trabalho de parto, os diagnósticos identificam as necessidades intrínsecas das parturientes, devendo ser o foco das ações do enfermeiro nesse período. Utilizou-se a 


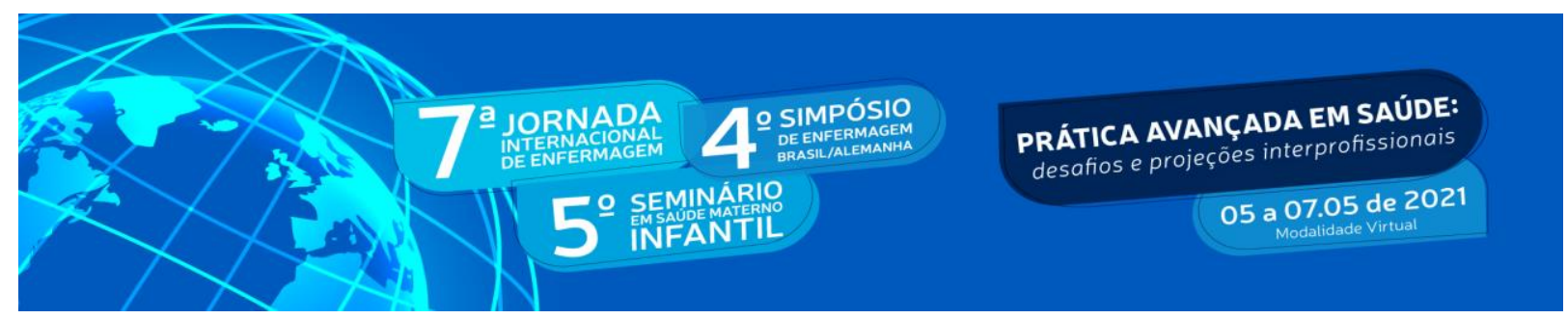

NANDA-I como referência para clasificação, e relacionou-se aos parâmetros emocinais, ambientais e funcionais, conforme modelo assistencial de Horta. Foi identificado que as intervenções não estão articuladas aos diagnósticos, e necessitam ser revisada.

Para COFEN (2009), o PE direciona a assistência dos profissionais de enfermagem e auxilia para a documentação das atividades desenvolvidas e orientadas por meio de embasamento teórico-científico, tais ações contribuem e refletem para uma maior notoriedade e valorização da profissão.

A SAE, regulada por meio da Lei do Exercício Profissional no 7489 de 25 de junho de 1986, tem como objetivo realizar ações específicas para promoção, prevenção, recuperação e reabilitação da saúde do sujeito e das pessoas ao seu redor, a SAE é responsável pela operacionalização do PE (COREN, 2016). O PE caracteriza-se por ações dinâmicas que tem como finalidade prestar assistência às pessoas, por meio de atos que se relacionam e coordenam entre si (HORTA, 1979).

Foi identificado na pesquisa de Azevedo et al. (2019) que o centro obstétrico faz parte dos setores onde menos ocorre a documentação no prontuário dos pacientes. Sendo 4,2 a média do número de fases que são documentadas no $\mathrm{CO}$ e em uma situação não foi realizado nenhum registro no prontuário. Reforçando que os locais onde não ficam pacientes internados acabam documentando menos, apesar da importância de documentar devido ao nível de complexidade dos atendimentos, e meia de garantir que ocorra a continuidade da assitência e promover segurança aos envolvidos. Além de estar descumprindo a lei, a falta do registro faz com que a atução do enfermeiro seja menos manifesta. Ante ao exposto, o estudo ressalta a importância não só da quantidade, mas também da qualidade dos registros.

A SAE é vista pelos enfermeiros como uma forma de enriquecer a assitência prestada às gestantes, realizando de forma sintetizada e organizada, executando todas as etapas necessárias ao exercício da enfermagem. Proporcionando autonomia ao enfermeiro, por aprofundar o conhecimento formal e assim proporcionando visibilidade aos profissionais (MEDEIROS; SANTOS; CABRAL, 2013).

$\mathrm{O}$ enfermeiro exerce um papel essencial no cuidado às gestantes, parturientes e puérperas. Por este motivo, torna-se importante a realização da SAE como rotina em setores com esse público específico. A gestação é um período muito particular na vida das mulheres, 


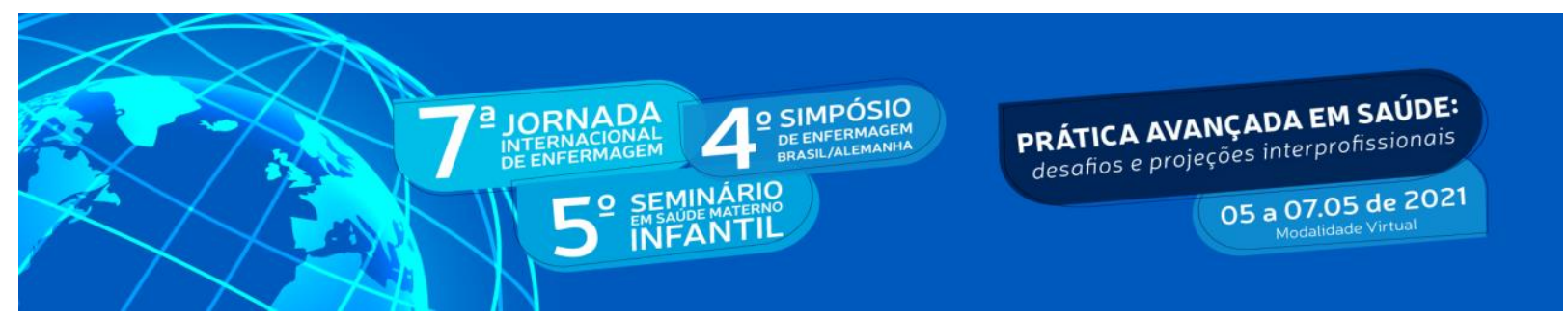

marcado por diversas modificações físicas, fisiológicas, psicossociais, mudanças que acabam gerando medos, dúvidas e anseios nas gestantes (ARAUJO, 2012). Essas sensações vividas pelas mulheres podem influenciar positiva ou negativamente o processo de gestar. Santos e Ramos (2012, p. 16), trazem em seu estudo algumas particularidades encontradas em pacientes no centro obstétrico, como: "medo do trabalho de parto, fadiga presente, contração uterina normal e dor de trabalho de parto presente.", além das intercorrências que podem ocorrer durante o parto em si e os medos vivenciados pelas parturientes.

Santos e Ramos (2012) reconhecem que o enfermeiro obstetra possui uma visão holística que auxilia na assistência individual à mulher em trabalho de parto, e associada ao processo de enfermagem proporciona um atendimento baseado em conhecimento científico, tornando o cuidado mais rico e natural, conforme o ritmo corporal da gestante.

Importante lembrar que em CO também ocorrem cesarianas, tendo em vista que o risco de infecção é um dado importante a ser observado, nos casos de cesárea, Silva et al. (2018, p. 3225) destaca o diagnóstico de risco de infecção, ressaltando "a infecção pós-parto como um processo infeccioso, considerando não somente a incisão cirúrgica, mas, também, as infecções uterinas, as complicações mamárias e respiratórias, as tromboflebites e as infecções urinárias".

Nota-se a importância de realizar o PE em centro obstétrico, tema ressaltado em uma pesquisa realizada por Santos e Ramos (2012), em um centro obstétrico que tinha como objetivo criar um protocolo para SAE voltado às parturientes de um hospital. A intenção dos pesquisadores era que o protocolo auxilie o enfermeiro a apresentar melhores resultados na sua assistência, por utilizar algo unificado e de acordo com a terminologia padrão.

Os profissionais de saúde reconhecem a importância do PE em sua assistência, porém, há diversos entraves para seu desempenho. Dias e Duran (2018) expõem em seu estudo algumas situações apontadas pelos enfermeiros que dificultam a implementação do PE, tais como, pouco contato com a temática durante a academia, falta de apoio da gestão, escassez de treinamentos ou realização de praticas pedagógicas relacionadas ao tema. Outros estudos também apontam o déficit de pessoal, a sobrecarga de trabalho e pouca experiência de trabalho como algumas das limitações encontradas pelos profissionais na prática do PE (DIAS; DURAN, 2018). 


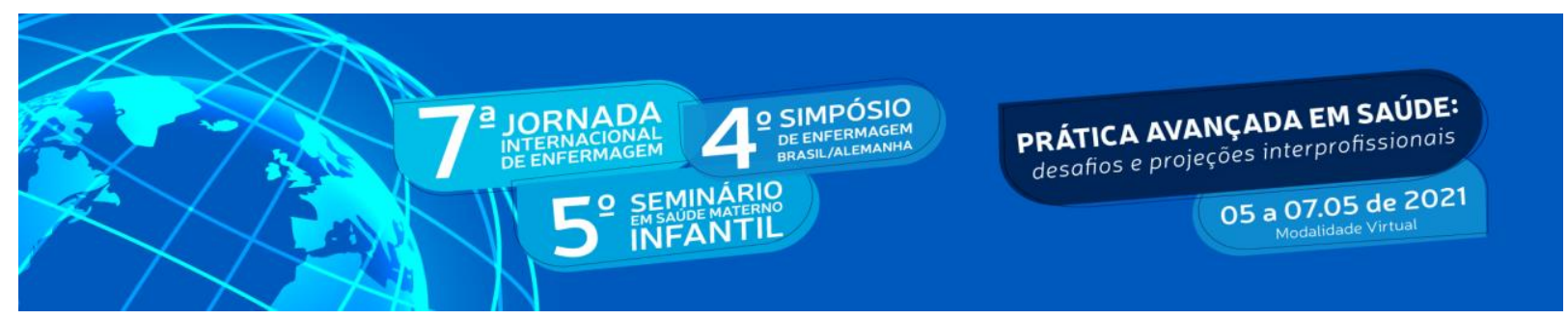

Algumas intervenções foram propostas pelos enfermeiros no estudo de Dias e Duran (2018), dentre elas, a realização de educação continuada pelos serviços de saúde, grupos de estudos referente ao tema e pesquisas para que haja visibilidade às experiências vivenciadas pelos profissionais que obtiveram sucesso na pratica do PE. A abordagem do PE em especializações, mestrado e doutorado, também foi apontada pelas participantes da pesquisa como algo que pode facilitar a prática dos profissionais.

A literatura apresenta diversos estudos que apontam a padronização da linguagem utilizada pelos pares, como algo importante e facilitador para o sucesso da implementação do PE, principalmente pela deficiência encontrada na academia e a prática profissional em relação ao tema.

\section{CONCLUSÃO}

O estudo mostrou as dificuldades para a utilização do PE em serviços de saúde, inclusive em $\mathrm{CO}$, sua implementação é importante por diversas razões, entre elas está a identificação e prevenção do risco de infecção em casos de cesarianas e outros procedimentos obstétrico. Além da proteção à paciente, o $\mathrm{PE}$ é importante em $\mathrm{CO}$ por proporcionar a melhora na assistência prestada às gestantes e puérperas por meio da padronização do atendimento e registros.

Foi evidenciado, nos artigos, o reconhecimento dos profissionais acerca do enriquecimento do PE na assistência prestada por eles, entretando, foi ressaltado que há fatores que dificultam sua realização da forma correta, entre eles está a falta de conhecimento e familiarização com o assunto, desde a graduação e menos ainda na atuação profissional, a falta de apoio da gestão e a inexistência de treinamentos. A sobrecarga no trabalho, o defict de funcionários e a pouca experiência de atuação foram apontados como outros fatores que dificultam a aplicação do PE.

Por fim, os enfermeiros apontaram como sugestões para a melhora da assistência e da implementação do PE algumas intervenções, dentre elas estão, grupos de estudo, treinaments, troca de experiências entre os profissionais, estudos de caso nos serviços de saúde. E na acadêmia, foi ressaltado que deve ser mais abordado o tema em cursos de especialização, 


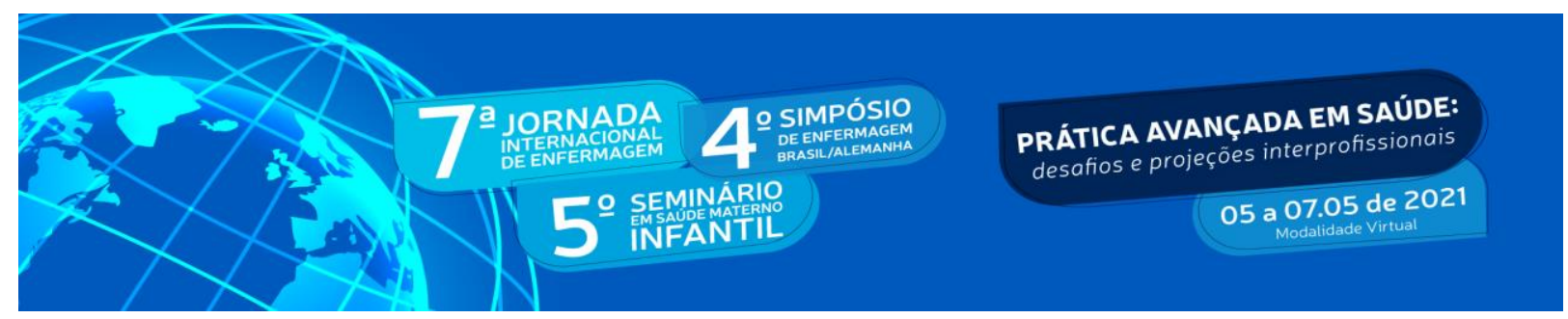

mestrado e doutorado.

\section{REFERÊNCIAS}

ARAUJO, N. M. et al. Corpo e sexualidade na gravidez. Rev. esc. enferm. USP [online]. 2012, vol.46, n.3, pp.552-558. ISSN 0080-6234. Disponível em: <https://www.scielo.br/pdf/reeusp/v46n3/04.pdf>. Acesso em: 08 set. 2020.

AZEVEDO O. A. et al. Documentation of the nursing process in public health institutions. Revista da Escola de Enfermagem USP, São Paulo, ed 53, 2019.

COFEN. 15 de outubro de 2009. RESOLUÇÃO COFEN-358/2009, Brasília-DF, 2009.

COREN - BA et al. SAE - Sistematização da assistência de enfermagem: UM GUIA PARA A PRÁTICA. Salvador, 2016, 40 p.

DIAS, L. B.; DURAN, E. C. M. Atitudes dos enfermeiros frente ao Processo de Enfermagem de um hospital público: estudo descritivo. Revista de enfermagem UERJ, Rio de Janeiro, v. 26, ed. 5, 2018.

MEDEIROS A. L.; SANTOS S. R.; CABRAL R. W. L., Desvelando dificuldades operacionais na sistematização da assistência de enfermagem através da Grounded Theory. Revista Eletrônica De Enfermagem, v. 15, ed. 1, 2013. p. 44-53.

MEDEIROS A. L. et al. Avaliando diagnósticos e intervenções de enfermagem no trabalho de parto e na gestação de risco. Revista Gaúcha de Enfermagem, RS, v. 37, ed. 3, 2016.

HORTA, W. de A.; com colaboração de CASTELLANOS, B. E. P. Processo de enfermagem: Guia prático. São Paulo: E.P.U. - Editora Pedagógica e Universitária Lida, 1979. $99 \mathrm{p}$.

SANTOS, R. B. dos; RAMOS, K, da S. Sistematização da assistência de enfermagem em Centro Obstétrico. Revista Brasileira de enfermagem, Recife, v. 65, ed. 1, p. 13-18, 2012.

SILVA, M. R. da et al. DIAGNÓSTICOS, RESULTADOS E INTERVENÇÕES DE ENFERMAGEM NO PARTO CESÁREO. Revista de enfermagem UFPE online, Recife, v. 12, n. 1981-8963, ed. 12, 2018. Disponível em: <https://periodicos.ufpe.br/revistas/revistaenfermagem/article/view/237549/30767>. Acesso em: 08 set. 2020 